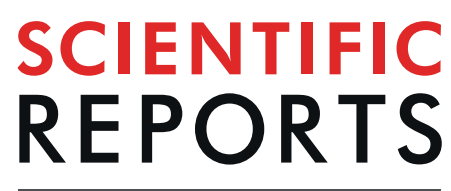

natureresearch

\title{
Acute pancreatitis promotes the generation of two different exosome populations
}

\begin{abstract}
A. Jiménez-Alesanco ${ }^{1,5}$, M. Marcuello ${ }^{2,5}$, M. Pastor-Jiménez ${ }^{1}$, L. López-Puerto ${ }^{1}$, L. Bonjoch ${ }^{1}$, M. Gironella $\mathbb{1}^{2}$, M. Carrascal ${ }^{3}$, J. Abian ${ }^{3}$, E. de-Madaria $\mathbb{1}^{4}$ \& D. Closa ${ }^{1 *}$

Exosomes are small extracellular vesicles that act as intercellular messengers. Previous studies revealed that, during acute pancreatitis, circulating exosomes could reach the alveolar compartment and activate macrophages. However, proteomic analysis suggested that the most likely origin of these exosomes could be the liver instead of the pancreas. The present study aimed to characterize the exosomes released by pancreas to pancreatitis-associated ascitic fluid (PAAF) as well as those circulating in plasma in an experimental model of taurocholate-induced acute pancreatitis in rats. We provide evidence that during acute pancreatitis two different populations of exosomes are generated with relevant differences in cell distribution, protein and microRNA content as well as different implications in their physiological effects. During pancreatitis plasma exosomes, but not PAAF exosomes, are enriched in the inflammatory miR-155 and show low levels of miR-21 and miR-122. Mass spectrometry-based proteomic analysis showed that PAAF exosomes contains 10-30 fold higher loading of histones and ribosomal proteins compared to plasma exosomes. Finally, plasma exosomes have higher pro-inflammatory activity on macrophages than PAAF exosomes. These results confirm the generation of two different populations of exosomes during acute pancreatitis. Deep understanding of their specific functions will be necessary to use them as therapeutic targets at different stages of the disease.
\end{abstract}

Acute pancreatitis is a frequent illness, being the 3rd leading cause of hospitalization due to gastrointestinal disease $^{1}$. It is an inflammatory process of the pancreas that, in the severe forms, is related to systemic inflammatory response and organ failure ${ }^{2}$. Currently there is no specific treatment available for pancreatitis beyond supportive care $^{3}$. This fact points to an incomplete understanding of the pathological mechanisms involved in the disease. From the first description of acute pancreatitis in 1895, it has been evaluated a number of mediators potentially involved in the progression of the disease from the local pancreatic damage to systemic inflammation. Along these years, hydrolytic enzymes, hormones, oxygen free radicals, cytokines, bioactive lipids and virtually any known molecule that can be suspected to have a role in the process, have been analyzed ${ }^{4-7}$. Unfortunately, this knowledge has not turned into the development of useful pharmacological treatments for the management of the disease.

Some of the lost pieces of this puzzle involve those mediators that do not circulate in a soluble form but packaged in microvesicles, including extracellular vesicles and exosomes. Exosomes are small extracellular vesicles that range between $30-200 \mathrm{~nm}$ in diameter that act as intercellular messengers by transferring signaling molecules, including proteins, small RNAs and lipids, to target cells. A number of studies have provided evidence of their implication in the pathogenesis of several diseases, being cancer and inflammation the fields in which

\footnotetext{
${ }^{1}$ Department of Experimental Pathology, Institut d'Investigacions Biomèdiques de Barcelona, Consejo Superior de Investigaciones Científicas (IIBB-CSIC), Institut d'Investigacions Biomèdiques August Pi i Sunyer (IDIBAPS), Barcelona, Spain. ${ }^{2}$ Gastrointestinal \& Pancreatic Oncology Group, Centro de Investigación Biomédica en Red de Enfermedades Hepáticas y Digestivas (CIBERehd)-IDIBAPS-Hospital Clínic de Barcelona, Barcelona, Spain. ${ }^{3}$ Proteomics Facility, Institut d'Investigacions Biomèdiques de Barcelona (IIBB), Consejo Superior de Investigaciones Científicas/Universitat Autònoma de Barcelona (CSIC/UAB), Institut d'Investigacions Biomèdiques August Pi i Sunyer (IDIBAPS), Barcelona, Spain. ${ }^{4}$ Pancreatic Unit, Department of Gastroenterology, Hospital General Universitario de Alicante, Instituto de Investigación Sanitaria y Biomédica de Alicante (ISABIAL - Fundación FISABIO), Alicante, Spain. ${ }^{5}$ These authors contributed equally: A. Jiménez-Alesanco and M. Marcuello. *email: daniel.closa@iibb.csic.es
} 

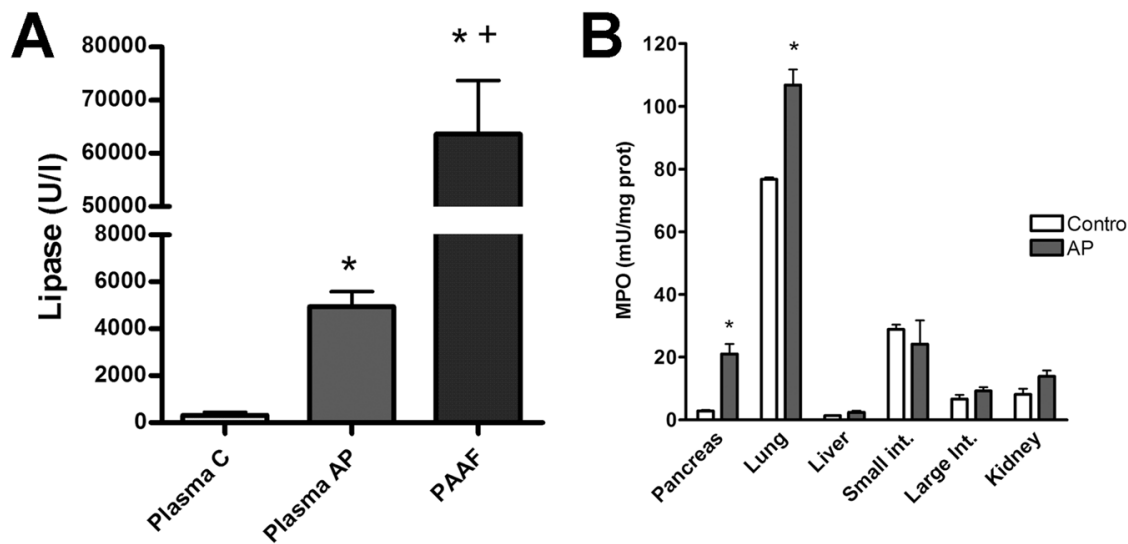

Figure 1. (A) Lipase in plasma from control (C) or acute pancreatitis (AP) animals and ascitic fluid (PAAF) and (B) myeloperoxidase (MPO) activity in different tissues $3 \mathrm{~h}$ after induction of acute pancreatitis. ${ }^{*} \mathrm{p}<0.05$ vs control; ${ }^{+} \mathrm{p}<0.05$ vs AP.

exosome research has more expanded the last years ${ }^{8}$. In particular, the role of exosomes in the progression of acute pancreatitis from the local damage to the systemic inflammation has been described ${ }^{9,10}$.

In previous studies, using an experimental model of taurocholate-induced acute pancreatitis in rats, we reported the generation and release to the bloodstream of a particular population of exosomes with the ability to reach and activate alveolar macrophages ${ }^{9}$. Interestingly, the proteomic analysis of exosomes purified from plasma of rats with acute pancreatitis did not showed the presence of pancreatic proteins. By contrast, it revealed a significant amount of proteins of supposedly hepatic origin, including apolipoproteins, mannose-binding protein or hemoglobin subunits. These observations suggest that, during acute pancreatitis, liver could be the source of exosomes that activate the inflammatory response in the lung. On the other hand, although we identified in the ascitic fluid the presence of exosomes allegedly released by the pancreas, it still remains to determine its composition and its potential role in the systemic effects of acute pancreatitis.

Here we characterized the two different populations of exosomes generated in an experimental model of acute pancreatitis in rats. The first one, purified from ascitic fluid, is generated by the pancreas while the second population of exosomes, collected from plasma, appeared to be released by the liver. We observed that, in addition to remarkable differences in protein and micro-RNA (mi-R) content, these exosomes showed significant differences in body distribution as well as in their physiopathological roles.

\section{Results}

Acute pancreatitis promotes inflammation in pancreas and lung. Pancreatitis results in a significant increase in lipase activity in plasma and also induces the peritoneal accumulation of PAAF containing high amounts of lipase (Fig. 1A). In PAAF, lipase activity was tenfold higher than levels observed in plasma. Inflammation, measured as increases in MPO activity, was observed only in pancreas and lung while other analysed tissues (Liver, kidney, small intestine and large intestine) showed no significant changes (Fig. 1B).

Exosomes in plasma and PAAF during acute pancreatitis. We have taken advantage of the fact that, in the experimental model of taurocholate-induced acute pancreatitis in rats, mediators released by the pancreas can be collected from PAAF. This allows us to analyse separately the exosomes coming from the pancreas and those that are circulating in the blood which are generated by other organs such as the liver. Extracellular vesicles isolated from both plasma and PAAF were morphologically characterized by transmission electron microscopy (TEM). The majority of vesicles had the classical exosome appearance of spherical bubbles or "cups" with the characteristic central depression, an artifact attributed to TEM sample preparation ${ }^{11}$. The observed size was around $100 \mathrm{~nm}$, as expected in the exosomes (Fig. 2A). This was confirmed by nanosight tracking analysis (Fig. 2B). The average of mode size of exosomes was $76.6 \pm 0.1 \mathrm{~nm}$ from plasma control; $91.6 \pm 5.0 \mathrm{~nm}$ for plasma from pancreatitis and $102.9 \pm 4.6 \mathrm{~nm}$ for PAAF, which agree with the size expected for exosomes. No significant differences were detected in total particle concentration and protein content (Fig. 2C).

Exosomes circulating in plasma are distributed to distant organs. To study the body distribution of circulating exosomes from plasma, vesicles obtained from control or pancreatitis animals were stained with PKH26 and injected into the cava vein of control rats. Thirty minutes later the presence of red fluorescent signal was detected in liver, lung and intestine. By contrast, they were not detected neither in the kidney nor pancreas. Noteworthy, there were no differences in organ distribution between control and AP exosomes (Fig. 3).

Hepatic capture of PAAF exosomes. As we have previously reported, PAAF exosomes stained with PKH26 red fluorescent dye and perfused through the portal vein were retained by the liver and uptaken by hepatocytes (Fig. 4A). We also compared, in hepatocytes cultured in vitro, the uptake of exosomes obtained from plasma or PAAF. Results indicate that hepatocytes incorporated significantly higher amount of exosomes when they came 

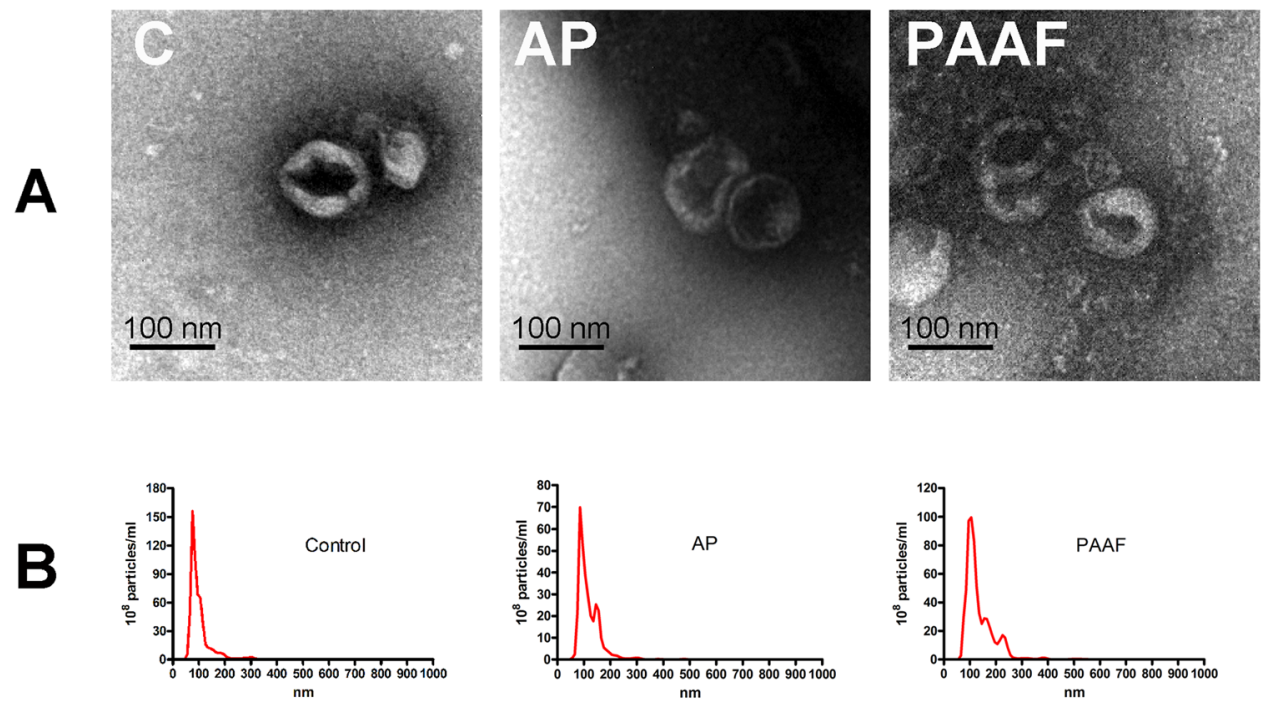

\begin{tabular}{c|c|c|c|c|}
\cline { 2 - 5 } & $\begin{array}{c}\text { Particles } / \mathrm{ml} \\
(\text { mean } \pm \text { s.e.m. }\end{array}$ & $\begin{array}{c}\text { Protein } \\
(\mathrm{mg} / \mathrm{ml})\end{array}$ & $\begin{array}{c}\text { Size } \\
(\text { Mean } \pm \text { s.e.m. })\end{array}$ & $\begin{array}{c}\text { Size } \\
(\text { Mode } \pm \text { s.e.m. })\end{array}$ \\
\hline Plasma Control & $5.83 \times 10^{9} \pm 1.50 \times 10^{8}$ & $8.3 \pm 1.7$ & $100.7 \pm 1.2$ & $76.6 \pm 0.1$ \\
\hline Plasma AP & $5.45 \times 10^{9} \pm 2.90 \times 10^{8}$ & $6.3 \pm 1.0$ & $117.5 \pm 2.2$ & $91.6 \pm 5.0$ \\
\hline PAAF & $6.60 \times 10^{9} \pm 3.29 \times 10^{8}$ & $9.6 \pm 1.4$ & $135.3 \pm 2.6$ & $102.9 \pm 4.6$ \\
\cline { 2 - 5 }
\end{tabular}

Figure 2. (A) Representative electron microscopy images of exosomes purified from plasma control (C), plasma from pancreatitis (AP) and Pancreatitis associated ascitic fluid (PAAF). (B) Nanovesicle tracking assay of extracellular vesicles. (C) Total number, protein concentration and size of particles obtained in plasma control, pancreatitis and PAAF.

from PAAF in comparison to the level of uptake observed when the exosomes were obtained from plasma control or plasma pancreatitis (Fig. 4B).

PAAF-exosomes and plasma-exosomes carry different miRNAs. Since the transfer of miRNA between cells is emerging as one of the main functions of exosomes, we evaluated the differences in the levels of three miRNAs in PAAF exosomes and plasma exosomes. The analysis revealed that after induction of acute pancreatitis, plasma exosomes contained increased amounts of the pro-inflammatory miR-155 and reduced levels of miR-122 and miR-21 in comparison to exosomes from plasma control. On the other hand, the levels of these miRNAs in PAAF exosomes were similar to that observed in plasma control (Fig. 5).

Proteomic analysis reveals differences in exosome cargo from plasma and PAAF. To evaluate the differences in exosome protein cargo between plasma and PAAF during acute pancreatitis, a mass spectrometry-driven proteomics analysis was performed. A total of 151 proteins were identified with high confidence, including CD81, commonly found in exosomes (Supplementary Table S1). The Gene Ontology Annotation term "extracellular exosome" was found significantly overrepresented in this collection where up to $70 \%$ of the proteins were annotated under this cellular compartment (Supplementary Table S2).

The comparative analysis showed that after pancreatitis induction exosomes in plasma were enriched in a number of proteins presumably expressed by the liver (Apolipoproteins, C-reactive protein, Retinol binding protein, Alpha-2-macroglobulin...) (Table 1). Moreover, only two specific pancreatic proteins were detected (Chimotrypsinogen $>2.2$ fold and amylase $>1.9$ fold). On the other hand, exosomes obtained from PAAF showed a completely different protein profile. In this case, relevant enrichment ( $>10$ fold) of several pancreatic proteins (Trypsin, Chymotrypsinogen, amylase, pancreatic elastase...) was detected but the higher enrichment was observed in histones ( $>30$ fold) and ribosomal proteins ( $>20$ fold) (Table 2).

Cellular responses to exosomes generated during acute pancreatitis. Since PAAF exosomes are retained in the liver while the plasma exosomes are distributed throughout the rest of the body and, moreover, they carry different content, we have analyzed whether there are also differences in the effects they have on two cell populations: alveolar macrophages and hepatocytes. In alveolar macrophages all treatments results in a small activation, however, the expression of the inflammatory cytokine IL- $1 \beta$ and chemokines CCL2 and CXCL1 was highly increased in response to exosomes from plasma AP. By contrast, exosomes from PAAF induces only a moderate increase that does not achieve statistical significance compared to control exosomes (Fig. 6A). On the other hand, treatment of hepatocytes with exosomes from any source did not result in relevant changes in the 


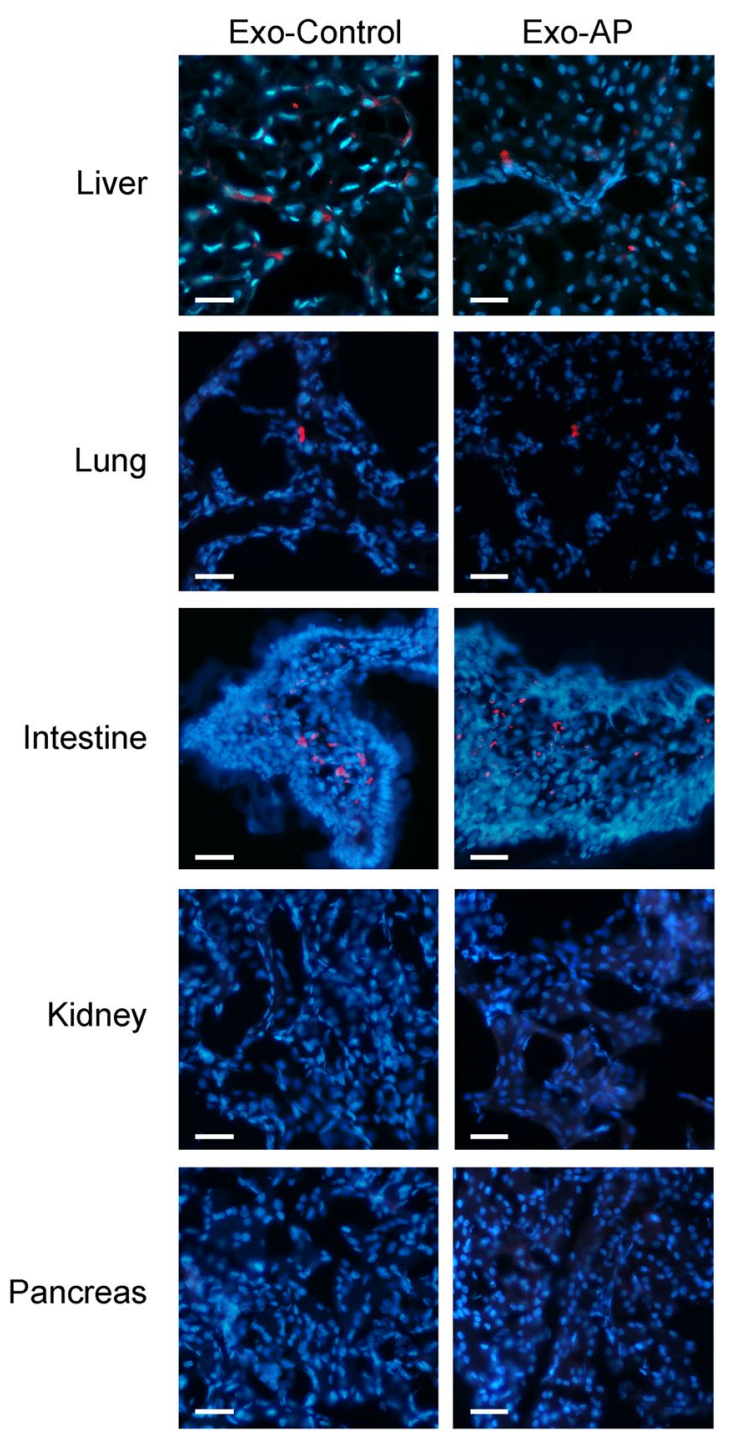

Figure 3. Exosomes obtained from Control and AP plasma samples were stained with PKH-26 dye (red) and perfused through the inferior vena cava. Tissue samples were obtained $30 \mathrm{~min}$ after administration. Nuclei were stained with DAPI (blue) (Scale bar: $50 \mu \mathrm{m})$.

mRNA expression of inflammation-related genes. No changes in IL-6, LBP or 11ßHSD1 expression were detected and only a moderate increase in $11 \beta \mathrm{HSD} 2$ expression was detected with PAAF exosomes (Fig. 6B).

\section{Discussion}

Exosomes have been shown to participate in inflammatory diseases by mediating antigen presentation and carrying different proteins and miRNAs. In the case of acute pancreatitis, the role of exosomes in activation of macrophages has been previously reported in vitro, by evaluating the role of exosomes released by stimulated pancreatic acinar cells on macrophages ${ }^{10}$, as well as in vivo, by using the taurocholate-induced pancreatitis model in rats $^{9}$. An unexpected conclusion of this study was the fact that the mechanisms involving the generation of these extracellular vesicles seems to be not restricted to the pancreas. Proteomic analysis of exosomes obtained from plasma in rats after induction of acute pancreatitis revealed the presence of different proteins of suspected hepatic origin and a lack of proteins with obvious pancreatic origin, pointing to the liver as a main source of the circulating exosomes.

Here we confirmed this possibility by comparing the content, distribution and physiological effects of exosomes obtained from plasma with those present in ascitic fluid of rats after induction of pancreatitis. In human patients ascites is not a frequent complication in acute pancreatitis; inflammatory exudates tend to form collections (liquid collections if necrosis is absent or necrotic collections in case of pancreatic and/or peripancreatic fat necrosis $)^{2}$ but in the experimental models in rats the pancreatic exudate is released to the peritoneum and could be easily recovered. This PAAF contains high concentrations of pancreatic enzymes and also extracellular vesicles, including exosomes, released by the pancreatic cells. We took advantage of this to compare pancreatic exosomes, obtained from ascitic fluid, with circulating plasma exosomes, supposedly released by the liver. 

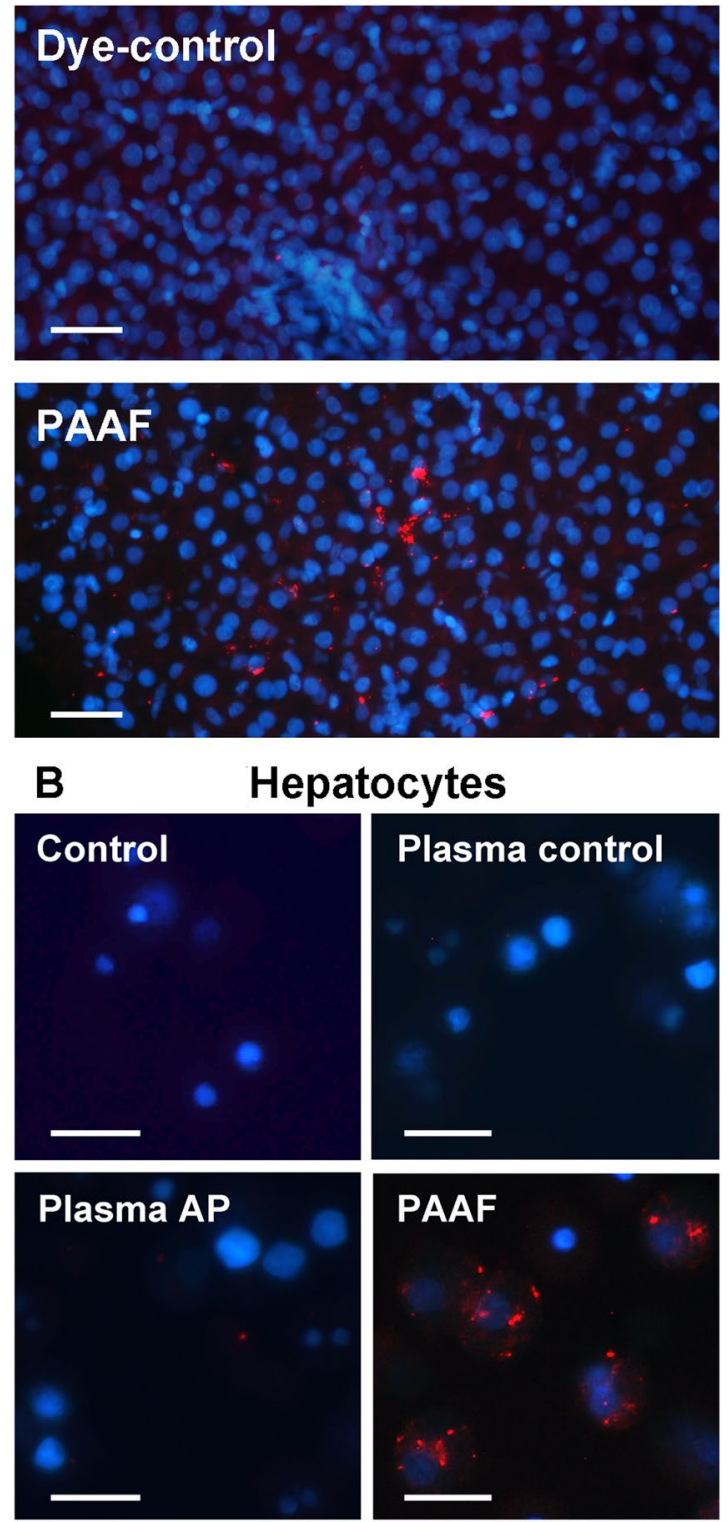

Figure 4. (A) Exosomes from PAAF were stained with PKH-26 dye (red) and perfused through the portal vein. PBS stained with PKH26 has been infused as a Dye-control group. Samples were obtained 30 min after administration. Nuclei were stained with DAPI (blue) (Scale: $50 \mu \mathrm{m})$. (B) Isolated hepatocytes were incubated in vitro for $30 \mathrm{~min}$. with exosomes (red) from plasma control, plasma AP or PAAF. Control group was incubated with PBS stained with PKH26 (Scale: $20 \mu \mathrm{m})$.
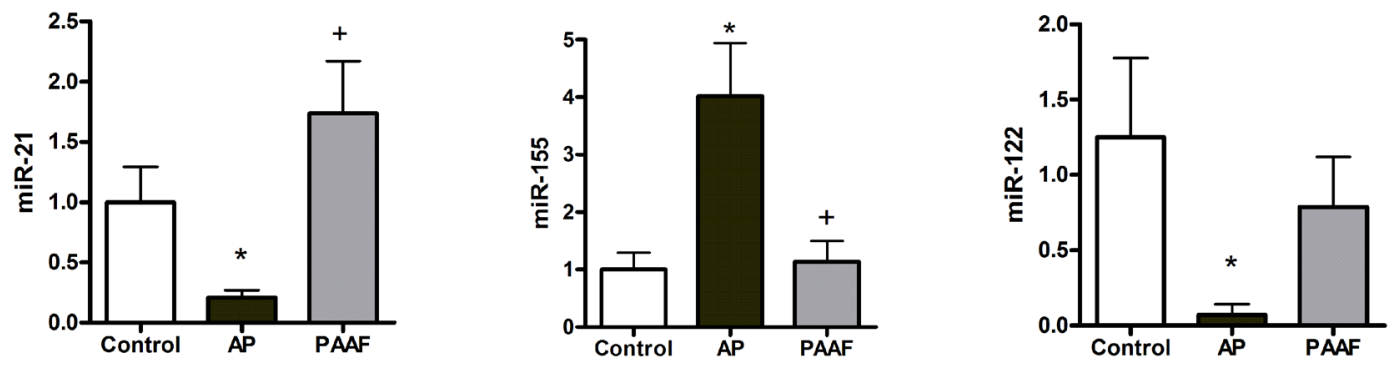

Figure 5. After induction of acute pancreatitis, exosomes from plasma transport higher levels of miR-155 and lower levels of miR-21 and miR-122. By contrast, no differences in those miRNAs were observed when comparing exosomes from PAAF and from plasma control. ${ }^{*} p<0.05$ vs control; ${ }^{+} p<0.05$ vs AP. 


\begin{tabular}{|c|c|c|c|}
\hline Protein ID & Protein name & Ratio & $p$-value \\
\hline P01946 & Hemoglobin subunit alpha-1/2 & 4.00 & $0.00 \mathrm{E}+00$ \\
\hline P02091 & Hemoglobin subunit beta- 1 & 2.88 & $1.39 \mathrm{E}-18$ \\
\hline P23562 & Band 3 anion transport protein & 2.66 & $1.90 \mathrm{E}-03$ \\
\hline P07338 & Chymotrypsinogen B (EC 3.4.21.1) & 2.28 & $2.31 \mathrm{E}-05$ \\
\hline P08932 & T-kininogen 2 & 2.21 & $1.22 \mathrm{E}-07$ \\
\hline P09006 & Serine protease inhibitor A3N (Serpin A3N) & 2.12 & $2.10 \mathrm{E}-66$ \\
\hline P11517 & Hemoglobin subunit beta- 2 & 2.08 & $4.45 \mathrm{E}-27$ \\
\hline P04785 & Protein disulfide-isomerase (EC 5.3.4.1) & 2.07 & $2.99 \mathrm{E}-03$ \\
\hline P00689 & Pancreatic alpha-amylase (EC 3.2.1.1) & 1.95 & $2.66 \mathrm{E}-04$ \\
\hline P02764 & Alpha-1-acid glycoprotein & 1.66 & $3.31 \mathrm{E}-17$ \\
\hline P02761 & Major urinary protein & 1.64 & $6.62 \mathrm{E}-05$ \\
\hline P01015 & Angiotensinogen (Serpin A8) & 1.53 & $8.04 \mathrm{E}-04$ \\
\hline P48199 & C-reactive protein & 1.52 & $5.73 \mathrm{E}-15$ \\
\hline P01048 & T-kininogen 1 & 1.45 & $3.99 \mathrm{E}-02$ \\
\hline P06238 & Alpha-2-macroglobulin & 1.43 & $6.20 \mathrm{E}-03$ \\
\hline P23680 & Serum amyloid P-component & 1.43 & $1.61 \mathrm{E}-04$ \\
\hline P04916 & Retinol-binding protein 4 & 1.36 & $2.13 \mathrm{E}-02$ \\
\hline P02650 & Apolipoprotein E (Apo-E) & 1.33 & $2.17 \mathrm{E}-11$ \\
\hline P14630 & Apolipoprotein M (Apo-M) & 1.32 & $1.27 \mathrm{E}-02$ \\
\hline P02767 & Transthyretin & 1.24 & $3.65 \mathrm{E}-11$ \\
\hline P04937 & Fibronectin & 1.23 & $4.72 \mathrm{E}-05$ \\
\hline P17475 & Alpha-1-antiproteinase (Alpha-1-antitrypsin) & 1.21 & $1.65 \mathrm{E}-64$ \\
\hline P04276 & Vitamin D-binding protein & 1.20 & $6.85 \mathrm{E}-12$ \\
\hline P10959 & Carboxylesterase 1C (EC 3.1.1.1) & 1.19 & $7.14 \mathrm{E}-09$ \\
\hline P36953 & Afamin & 1.14 & $4.76 \mathrm{E}-05$ \\
\hline Q9WUW3 & Complement factor I (EC 3.4.21.45) & 1.13 & $3.99 \mathrm{E}-02$ \\
\hline P05545 & Serine protease inhibitor A3K (Serpin A3K) & 1.12 & $7.37 \mathrm{E}-04$ \\
\hline P14480 & Fibrinogen beta chain & 1.10 & $3.21 \mathrm{E}-04$ \\
\hline P20059 & Hemopexin & 1.08 & $1.65 \mathrm{E}-06$ \\
\hline P05544 & Serine protease inhibitor A3L (Serpin A3L) & 1.08 & $1.65 \mathrm{E}-06$ \\
\hline P31211 & Corticosteroid-binding globulin (CBG) & 1.08 & $2.68 \mathrm{E}-02$ \\
\hline P06399 & Fibrinogen alpha chain & 1.07 & $2.29 \mathrm{E}-02$ \\
\hline P02770 & Serum albumin & 1.05 & $1.28 \mathrm{E}-10$ \\
\hline Q03626 & Murinoglobulin-1 & 1.03 & $3.96 \mathrm{E}-03$ \\
\hline P12346 & Serotransferrin & 1.02 & $1.45 \mathrm{E}-02$ \\
\hline P01026 & Complement C3 & 0.97 & $1.52 \mathrm{E}-04$ \\
\hline P14046 & Alpha-1-inhibitor 3 & 0.95 & $5.03 \mathrm{E}-05$ \\
\hline P01835 & Ig kappa chain $\mathrm{C}$ region, $\mathrm{B}$ allele & 0.92 & $1.65 \mathrm{E}-02$ \\
\hline Q99PS8 & Histidine-rich glycoprotein & 0.89 & $2.04 \mathrm{E}-03$ \\
\hline Q62862 & MAP kinase kinase 5 (MAPKK 5) (EC 2.7.12.2) & 0.84 & $4.30 \mathrm{E}-03$ \\
\hline P20762 & Ig gamma-2C chain $\mathrm{C}$ region & 0.81 & $1.85 \mathrm{E}-04$ \\
\hline Q6P6T1 & Complement C1s subcomponent (EC 3.4.21.42) & 0.69 & $4.04 \mathrm{E}-02$ \\
\hline Q63041 & Alpha-1-macroglobulin & 0.68 & $5.15 \mathrm{E}-167$ \\
\hline P02651 & Apolipoprotein A-IV (Apo-AIV) & 0.64 & $3.07 \mathrm{E}-10$ \\
\hline P06866 & Haptoglobin & 0.27 & $2.32 \mathrm{E}-97$ \\
\hline
\end{tabular}

Table 1. Differentially expressed proteins found between exosomes obtained from plasma AP respect to plasma $\mathrm{C} 3 \mathrm{~h}$ after induction of acute pancreatitis.

In a previous work ${ }^{9}$ we observed that, due to the anatomical situation of the pancreas, vesicles released by this organ are collected into the portal system and circulate through the liver before reaching the systemic circulation in the vena cava. This fact is relevant for the final fate of exosomes released by the pancreas and our data confirm that they are retained in the liver (Fig. 4A). However, when we compared the affinity of the hepatocytes for the vesicles obtained from PAAF with those obtained from plasma, we observed that the absorption of pancreatic exosomes was much higher than the absorption of exosomes circulating in plasma (Fig. 4B). This difference already suggests that the two populations of vesicles present significant differences in their composition.

On the other hand, tracking experiment of exosomes obtained from plasma and injected into the cava vein revealed that they are distributed in different organs and they do not show any particular affinity for the liver (Fig. 3). In addition, there are no evident differences between the exosomes obtained from control animals than 


\begin{tabular}{|c|c|c|c|}
\hline Protein ID & Protein name & Ratio & $p$-value \\
\hline A9UMV8 & Histone H2A.J & 30.67 & 0.005677 \\
\hline Q00715 & Histone $\mathrm{H} 2 \mathrm{~B}$ & 24.74 & $2.22 \mathrm{E}-25$ \\
\hline P63245 & Receptor of activated protein C kinase 1 & 23.27 & 0.047568 \\
\hline P60868 & 40S ribosomal protein $\mathrm{S} 20$ & 21.79 & 0.001606 \\
\hline P62804 & Histone H4 & 17.50 & $1.94 \mathrm{E}-24$ \\
\hline P00773 & Chymotrypsin-like elastase 1 (EC 3.4.21.36) & 16.94 & $4.65 \mathrm{E}-11$ \\
\hline P04797 & GAPDH (EC 1.2.1.12) & 15.50 & $2.3 \mathrm{E}-17$ \\
\hline P84245 & Histone $\mathrm{H} 3.3$ & 13.89 & 0.000248 \\
\hline P08426 & Cationic trypsin-3 (EC 3.4.21.4) & 12.85 & $5.68 \mathrm{E}-07$ \\
\hline P35427 & $60 \mathrm{~S}$ ribosomal protein $\mathrm{L} 13 \mathrm{a}$ & 12.83 & 0.001105 \\
\hline P02262 & Histone $\mathrm{H} 2 \mathrm{~A}$ type 1 & 12.75 & $2.01 \mathrm{E}-09$ \\
\hline P07338 & Chymotrypsinogen B (EC 3.4.21.1) & 12.08 & $1.89 \mathrm{E}-14$ \\
\hline P62083 & 40 ribosomal protein S7 (S8) & 11.37 & $6.49 \mathrm{E}-06$ \\
\hline P05197 & Elongation factor 2 (EF-2) & 11.32 & $6.65 \mathrm{E}-09$ \\
\hline Q5XIF6 & Tubulin alpha-4A chain & 11.29 & 0.000617 \\
\hline P62630 & Elongation factor 1-alpha 1 & 11.13 & $1.32 \mathrm{E}-28$ \\
\hline P19223 & Carboxypeptidase B (EC 3.4.17.2) & 10.73 & $1.83 \mathrm{E}-29$ \\
\hline P00689 & Pancreatic alpha-amylase (PA) (EC 3.2.1.1) & 9.64 & $8.75 \mathrm{E}-26$ \\
\hline P00564 & Creatine kinase M-type (EC 2.7.3.2) & 8.89 & $2.66 \mathrm{E}-10$ \\
\hline P49242 & $40 \mathrm{~S}$ ribosomal protein $\mathrm{S} 3 \mathrm{a}$ & 6.90 & 0.014226 \\
\hline P62250 & 40S ribosomal protein S16 & 6.57 & 0.007414 \\
\hline P09812 & Glycogen phosphorylase (EC 2.4.1.1) & 6.09 & $3.57 \mathrm{E}-09$ \\
\hline P06761 & Endoplasmic reticulum chaperone (EC 3.6.4.10) & 5.69 & 0.000417 \\
\hline P04785 & Protein disulfide-isomerase (PDI) (EC 5.3.4.1) & 5.45 & $1.67 \mathrm{E}-06$ \\
\hline P00774 & Chymotrypsin-like elastase 2A (EC 3.4.21.71) & 5.12 & $2.79 \mathrm{E}-07$ \\
\hline P02091 & Hemoglobin subunit beta-1 (Beta-1-globin) & 4.74 & $5.38 \mathrm{E}-65$ \\
\hline P01946 & Hemoglobin subunit alpha-1/2 (Alpha-1/2-globin) & 4.23 & 0 \\
\hline P15083 & Polymeric immunoglobulin receptor & 3.99 & 0.00096 \\
\hline P63324 & 40S ribosomal protein $\mathrm{S} 12$ & 3.84 & 0.004548 \\
\hline P11517 & Hemoglobin subunit beta- 2 & 3.84 & $6.19 \mathrm{E}-96$ \\
\hline P05371 & Clusterin & 2.83 & $1.35 \mathrm{E}-16$ \\
\hline Q64268 & Heparin cofactor 2 (Serpin D1) & 2.81 & 0.023958 \\
\hline P62738 & Actin, aortic smooth muscle & 2.78 & 0.039368 \\
\hline Q62930 & Complement component C9 & 2.77 & $8.08 \mathrm{E}-09$ \\
\hline P06866 & Haptoglobin & 2.74 & $1.55 \mathrm{E}-67$ \\
\hline P23562 & Band 3 anion transport protein $(\mathrm{CD} 233)$ & 2.66 & $6.76 \mathrm{E}-05$ \\
\hline P08650 & Complement C5 & 2.42 & 0.000259 \\
\hline Q811M5 & Complement component C6 & 2.25 & 0.004227 \\
\hline P20759 & Ig gamma-1 chain $\mathrm{C}$ region & 2.13 & $2.84 \mathrm{E}-06$ \\
\hline P04916 & Retinol-binding protein 4 & 2.13 & $2.84 \mathrm{E}-06$ \\
\hline P55314 & Complement component $\mathrm{C} 8$ beta chain & 2.00 & 0.023921 \\
\hline Q01177 & Plasminogen (EC 3.4.21.7) & 1.97 & $1.6 \mathrm{E}-95$ \\
\hline Q68FP1 & Gelsolin & 1.93 & $5.27 \mathrm{E}-05$ \\
\hline Q7TMA5 & Apolipoprotein B-100 (Apo B-100) & 1.66 & 0.006874 \\
\hline P18292 & Prothrombin (EC 3.4.21.5) & 1.61 & $1.85 \mathrm{E}-09$ \\
\hline Q63416 & Inter-alpha-trypsin inhibitor heavy chain $\mathrm{H} 3$ & 1.59 & $2.84 \mathrm{E}-06$ \\
\hline Q6P6T1 & Complement C1s subcomponent (EC 3.4.21.42) & 1.58 & 0.011707 \\
\hline P24090 & Alpha-2-HS-glycoprotein (Fetuin-A) & 1.55 & $7.06 \mathrm{E}-45$ \\
\hline P08649 & Complement C4 & 1.53 & $1.43 \mathrm{E}-17$ \\
\hline P04937 & Fibronectin (FN) & 1.53 & $3.09 \mathrm{E}-17$ \\
\hline P14272 & Plasma kallikrein (EC 3.4.21.34) & 1.51 & 0.010077 \\
\hline P25236 & Selenoprotein $\mathrm{P}(\mathrm{SeP})$ & 1.50 & 0.00256 \\
\hline Q63556 & Serine protease inhibitor A3M (Serpin A3M) & 1.49 & $9.43 \mathrm{E}-06$ \\
\hline Q62862 & MAPKK 5 (EC 2.7.12.2) & 1.46 & $4.63 \mathrm{E}-07$ \\
\hline P02651 & Apolipoprotein A-IV (Apo-AIV) & 1.46 & $1.1 \mathrm{E}-07$ \\
\hline Q9QX79 & Fetuin-B & 1.45 & $1.23 \mathrm{E}-20$ \\
\hline
\end{tabular}




\begin{tabular}{|c|c|c|c|}
\hline Protein ID & Protein name & Ratio & $p$-value \\
\hline P55159 & $\begin{array}{l}\text { Serum paraoxonase/arylesterase 1 (PON 1) (EC 3.1.1.2) (EC } \\
\text { 3.1.1.81) (EC 3.1.8.1) }\end{array}$ & 1.42 & 0.020857 \\
\hline Q6P734 & Plasma protease $\mathrm{C} 1$ inhibitor (Serpin G1) & 1.36 & 0.005552 \\
\hline P05545 & Serine protease inhibitor A3K (Serpin A3K) & 1.32 & $4.31 \mathrm{E}-15$ \\
\hline Q63514 & C4b-binding protein alpha chain (C4bp) & 1.31 & 0.003327 \\
\hline P14630 & Apolipoprotein M (Apo-M) & 1.30 & 0.011425 \\
\hline P01015 & Angiotensinogen (Serpin A8) & 1.30 & 0.018252 \\
\hline P20761 & Ig gamma-2B chain $\mathrm{C}$ region & 1.30 & 0.001105 \\
\hline P14480 & Fibrinogen beta chain & 1.29 & $3.04 \mathrm{E}-21$ \\
\hline P01835 & Ig kappa chain $\mathrm{C}$ region, $\mathrm{B}$ allele & 1.29 & $4.95 \mathrm{E}-15$ \\
\hline P00762 & Anionic trypsin-1 (EC 3.4.21.4) & 1.25 & $6.49 \mathrm{E}-06$ \\
\hline Q64240 & Protein AMBP & 1.24 & 0.005677 \\
\hline P05544 & Serine protease inhibitor A3L (Serpin A3L) & 1.24 & $1.33 \mathrm{E}-35$ \\
\hline P20760 & Ig gamma-2A chain $\mathrm{C}$ region & 1.22 & 0.000398 \\
\hline P02680 & Fibrinogen gamma chain & 1.19 & $7.24 \mathrm{E}-09$ \\
\hline Q9WUW3 & Complement factor I (EC 3.4.21.45) & 1.17 & 0.007751 \\
\hline P02650 & Apolipoprotein E (Apo-E) & 1.14 & 0.001062 \\
\hline P20762 & Ig gamma-2C chain $\mathrm{C}$ region & 1.14 & 0.016552 \\
\hline P17475 & Alpha-1-antiproteinase (Alpha-1-antitrypsin) & 1.13 & $1.04 \mathrm{E}-29$ \\
\hline Q99PS8 & Histidine-rich glycoprotein & 1.09 & 0.025281 \\
\hline P02767 & Transthyretin (Prealbumin) & 1.09 & 0.00852 \\
\hline P20059 & Hemopexin & 0.96 & 0.015278 \\
\hline P36953 & Afamin (Alpha-albumin) & 0.94 & 0.035576 \\
\hline P12346 & Serotransferrin (Transferrin) & 0.92 & $8.05 \mathrm{E}-32$ \\
\hline Q63041 & Alpha-1-macroglobulin & 0.92 & $8.15 \mathrm{E}-42$ \\
\hline P01026 & Complement C3 & 0.90 & $6.76 \mathrm{E}-57$ \\
\hline P10959 & Carboxylesterase 1C (EC 3.1.1.1) & 0.89 & 0.000116 \\
\hline P06399 & Fibrinogen alpha chain & 0.78 & $3.53 \mathrm{E}-15$ \\
\hline P09006 & Serine protease inhibitor A3N (Serpin A3N) & 0.76 & $2.96 \mathrm{E}-14$ \\
\hline Q03626 & Murinoglobulin-1 & 0.72 & $1 \mathrm{E}-176$ \\
\hline P14046 & Alpha-1-inhibitor 3 & 0.71 & $3.2 \mathrm{E}-163$ \\
\hline P02764 & Alpha-1-acid glycoprotein (Orosomucoid) & 0.65 & $3.61 \mathrm{E}-14$ \\
\hline P08932 & T-kininogen 2 (Major acute phase protein) & 0.64 & 0.000121 \\
\hline P08934 & Kininogen-1 & 0.64 & 0.000579 \\
\hline P23764 & Glutathione peroxidase 3 (EC 1.11.1.9) & 0.63 & $4.82 \mathrm{E}-06$ \\
\hline P04639 & Apolipoprotein A-I (Apo-AI) & 0.58 & $8.21 \mathrm{E}-69$ \\
\hline P48199 & C-reactive protein & 0.45 & $5.56 \mathrm{E}-31$ \\
\hline P04638 & Apolipoprotein A-II (Apo-AII) & 0.37 & $2.07 \mathrm{E}-07$ \\
\hline P19939 & Apolipoprotein C-I (Apo-CI) & 0.27 & $7.07 \mathrm{E}-10$ \\
\hline
\end{tabular}

Table 2. Differentially expressed proteins found between exosomes obtained from PAAF respect to plasma AP $3 \mathrm{~h}$ after induction of acute pancreatitis.

those ones obtained after the induction of pancreatitis, suggesting that there are no specific organ targets for these vesicles. Other variables, as local blood flow, could be the most relevant factors affecting the distribution of exosomes in the systemic circulation.

However, the main difference between the two populations of exosomes can be seen by comparing their cargo. Comparison between exosomes from plasma control and pancreatitis revealed that pancreatitis results in an enrichment of miR-155, and a reduction of miR-122 and miR-21 (Fig. 5). These changes were not observed in PAAF exosomes, in which the levels of these miRNAs are closely similar to those observed in the exosomes from plasma control, thus reinforcing the idea that there are two different exosome populations generated during acute pancreatitis.

Although most miRNA are multifunctional, the pro-inflammatory role of miR-155 is well known and it has been shown to promote M1 polarization of macrophages ${ }^{12}$. By contrary, miR-122, which is mainly produced by the liver and secreted into bloodstream, appears to play a protective and anti-inflammatory role ${ }^{13,14}$. Reduction of miR-122 in blood is associated with inflammation in several diseases and its deletion was shown to drive microsteatosis and inflammation ${ }^{15}$. The case for miR-21 is more confusing and it has been reported to play a role both in increasing and in preventing cell damage ${ }^{16,17}$. However, the administration of miR-21 prevented the inflammatory activation of macrophages in several models ${ }^{18,19}$. Therefore, our results suggest that during acute pancreatitis exosomes circulating in plasma are enriched in pro-inflammatory (miR-155) exosomes and have reduced levels of other miRNAs (miR-21 and miR-122) which could play an anti-inflammatory response, 


\section{A Alveolar macrophages}
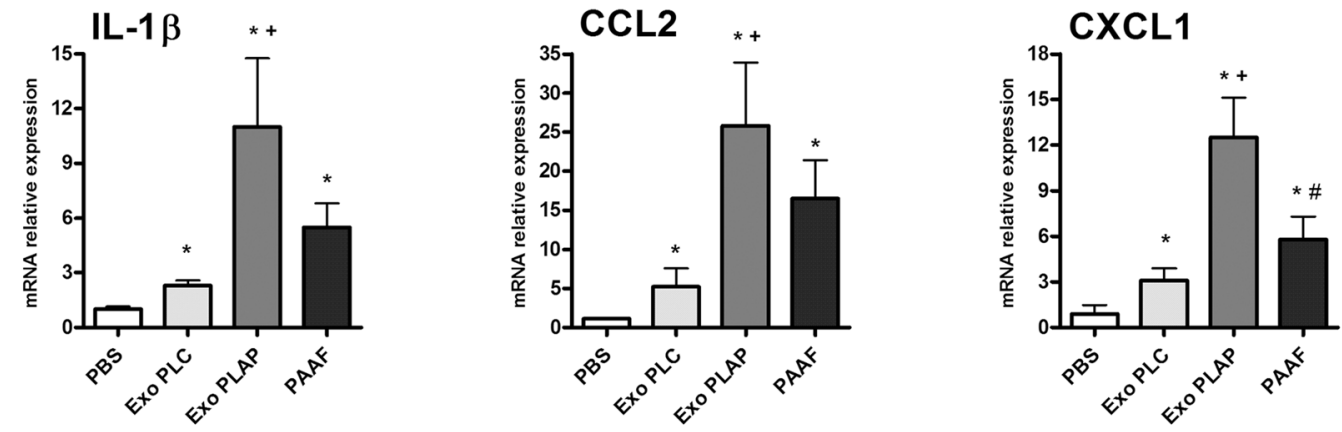

B

\section{Hepatocytes}
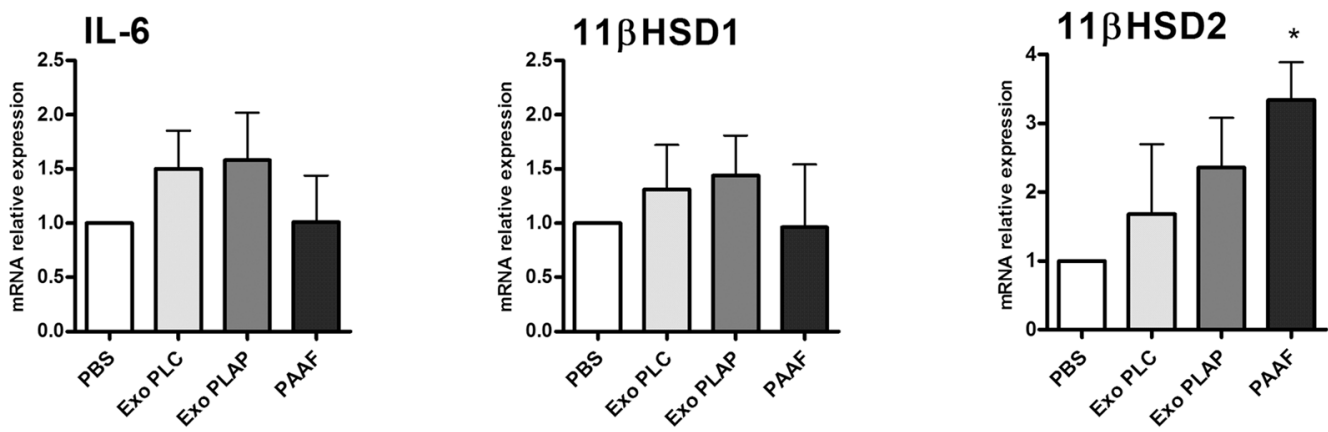

Figure 6. (A) Effect of exosomes $(2 \mu \mathrm{g} / \mathrm{ml})$ in macrophage activation. Even the exosomes from plasma control (exo PLC) results in a small activation of macrophages. However, exosomes from plasma AP (exo PLAP) induces higher induction of IL1 $\beta$, CCL2 and CXCL1. PAAF exosomes also increases the expression of these mediators but not achieves statistical significance compared to control plasma. Control cells are treated with the equivalent volume of PBS. (B) In hepatocytes the only effect observed was an increase in the expression of $11 \beta$ HSD2 under the treatment with PAAF exosomes. $* p<0.05$ vs control; ${ }^{+} p<0.05$ vs Exo PLC; $p<0.05$ vs Exo PLAP.

particularly in macrophages. This agree with the induction of systemic inflammatory conditions along the progression of severe forms of acute pancreatitis and could be related with the effect of these exosomes activating macrophages and promoting the release of inflammatory cytokines (Fig. 6A). Finally, it has been reported that inhibition of miR-155 improves prognosis in an experimental model of acute pancreatitis, making this miRNA a promising target for the design of therapeutical strategies for the treatment of pancreatitis ${ }^{20}$. Our results indicate that, in addition to the local synthesis, this miRNA could achieve the target macrophages via exosomes, providing a potential new point of control.

Even more marked are the differences in protein content (Table 1). As we have previously reported, exosomes from plasma AP are enriched in a number of hepatic proteins, including apolipoproteins, CRP or LBP when compared with exosomes from plasma control ${ }^{9}$. Now, the comparison between exosomes from plasma and PAAF in animals with pancreatitis revealed a completely different profile (Table 2). First, the fact that PAAF exosomes contains high levels of different pancreatic enzymes, including trypsin, amylase or chymotrypsinogen, confirms their pancreatic origin. On the other hand, the major increase detected in PAAF exosomes corresponds to some histones and ribosomal proteins, virtually absent in plasma exosomes. This fact suggests that PAAF exosomes, but not plasma exosomes, contain the nucleoprotein component TERRA (Telomeric repeat-containing RNA), a telomere-associated regulator of chromosome end protection that has been reported to be carried by exosomes from damaged cells and can elicit an inflammatory response ${ }^{21}$. This complex is made by a noncoding RNA transcript associated with histone proteins ${ }^{22}$.

We also evaluated the differences in the response of alveolar macrophages and hepatocytes to these two populations of exosomes. In alveolar macrophages the treatment with exosomes induces a response, reflected in increases in IL-1 $\beta$, CCL2 and CXCL1 expression in all conditions, even with control plasma exosomes. However, the response obtained by treating the cells with exosomes from plasma AP is clearly superior, thus reflecting the 
pro-inflammatory activity of these vesicles. By contrast, exosomes from PAAF only results in a moderate induction, which is not statistically different to those obtained with control plasma (Fig. 6A).

On the other hand, we evaluated in hepatocytes the effect of exosomes on the expression of IL- 6 and in the enzymes $11 \beta$-hydroxysteroid dehydrogenase type 1 (11ßHSD1) and type 2 (11ßHSD2). These enzymes were selected since they are involved in the liver metabolism of corticosterone and play a role in modulating the levels of circulating corticosterone, thus controlling the anti-inflammatory status of the organism $\mathrm{m}^{23,24}$ and are altered during acute pancreatitis ${ }^{25}$. The only effect observed was an increase in the expression of $11 \beta \mathrm{HSD} 2$ induced by PAAF exosomes while no changes were detected for IL-6 or 11ßHSD1 (Fig. 6B). Since 11ßHSD2 oxidizes the active corticosterone (in rats) or cortisol (in humans) to the inactive cortisone, an increase in the expression of this enzyme could be related with the impaired anti-inflammatory response of the organism that occurs during pancreatitis.

Altogether, our results confirms that during acute pancreatitis, there are generated two populations of exosomes, with clear differences in their origins, tissue distribution, molecular content and physiological effects Exosomes generated by the pancreas appear to act on the liver while exosomes circulating in plasma are generated by the liver and would act as pro-inflammatory mediators activating macrophages in lung and, probably, in other systemic organs. In light of the results obtained, both populations could be interesting potential therapeutic targets for different stages of the disease. Although further studies are required to validate these results in human patients, the absence of ascitic fluid in this case will made difficult to separate the two populations of exosomes. Anyway, we can hypothesize that an early selective or non-selective removal of pro-inflammatory exosomes from plasma is a promising treatment to decrease systemic inflammation and thus, the development of organ failure, condition that is associated to a high risk of mortality ${ }^{26}$.

\section{Methods}

Animals. Male Wistar rats (250 g b.w.) (Charles River, France) were housed in a controlled environment, fed with standard laboratory pelleted formula (A04, Panlab, Spain) and tap water ad libitum. This study conformed to European Community for the use of experimental animals and was approved by the Institutional Committee of Animal Care and Research: CEEA-UB (Comitè Ėtic d'Experimentació Animal de la Universitat de Barcelona), procedure number 10345 .

Animal model of acute pancreatitis. Animals ( $\mathrm{n}=6$ each group) were anesthetized with an i.p. administration of sodium pentobarbital $(50 \mathrm{mg} / \mathrm{kg})$. After midline laparotomy, biliopancreatic duct was cannulated through the duodenum and a small bulldog clamp was used to close the hepatic duct. AP was induced by retrograde perfusion of $5 \%$ sodium taurocholate in a volume of $0.1 \mathrm{ml} / 100 \mathrm{~g}$ b.w. using a perfusion pump (Harvard Instruments, Edenbridge, $\mathrm{UK})^{27}$. Control animals received saline solution $(\mathrm{NaCl} 0.9 \%)$. This is a very aggressive model for pancreatic tissue, but reproduces very well the systemic effects of pancreatitis. Three hours after induction, samples of blood and pancreatitis-associated ascitic fluid (PAAF) were collected and processed immediately for the isolation of exosomes. This time point was selected since at shorter induction times inflammation process in lung has not started ${ }^{28}$.

Lipase activity. Measurement of plasma and PAAF lipase activity was carried out by using the turbidimetric assay kit from Randox (Antrim, U.K.), according to the supplier's specifications.

Myeloperoxidase activity. Neutrophilic infiltration was analyzed by measuring myeloperoxidase (MPO) activity. Samples were homogenized with $0.5 \%$ hexadecyltrimethylammonium bromide in $50 \mathrm{mM} \mathrm{PBS}$ at pH 6.0. Homogenates were disrupted for $30 \mathrm{~s}$ using a Labsonic sonicator (Braun Biotech, Inc., Allentown, PA), submitted to three cycles of freezing and thawing and sonicated again. Samples were incubated at $60^{\circ} \mathrm{C}$ for $2 \mathrm{~h}$, centrifuged at $15,000 \times \mathrm{g}$ for $15 \mathrm{~min}$ and supernatants were collected for MPO assay and protein assay. MPO activity was determined photometrically at $655 \mathrm{~nm}$ using $\mathrm{H}_{2} \mathrm{O}_{2}$ and 3,3',5,5'-tetramethylbenzidine as substrates ${ }^{29}$.

RNA isolation and qPCR. Total RNA from cells was extracted using the TRizol ${ }^{\circledR}$ reagent (Invitrogen, Carlsbad, CA). RNA was quantified by measuring the absorbance at 260 and $280 \mathrm{~nm}$ using a NanoDrop ND-1000 spectrophotometer (NanoDrop Technologies, USA). cDNA was synthesized from $1 \mu \mathrm{g}$ RNA sample using the iScript cDNA synthesis kit (Bio-Rad, CA, USA).

Subsequent quantitative polymerase chain reaction (qPCR) was performed in a DNA Engine, Peltier Thermal Cycler (Bio-Rad, CA, USA) using iTaq ${ }^{\mathrm{TM}}$ Universal SYBR ${ }^{\circledR}$ Green Super mix and the corresponding rat primers (Supplementary Table S3). Reactions were performed in duplicate and threshold cycle values were normalized to GAPDH gene expression. The specificity of the products was determined by melting curve analysis. The relative expression of target genes to $G A P D H$ was calculated by the $\triangle \mathrm{C}(\mathrm{t})$ formula.

Isolation of hepatocytes and alveolar macrophages. Hepatocytes were isolated from control rats by collagenase digestion ${ }^{30}$, and cultured in 12 wells plates in $5 \% \mathrm{CO}_{2}$ in air at $37^{\circ} \mathrm{C}$ using Exo-Free supplemented DMEM medium (9\% FBS, $100 \mathrm{U} / \mathrm{ml}$ penicillin and $100 \mu \mathrm{g} / \mathrm{ml}$ streptomycin $)^{31}$.

To obtain alveolar macrophages, lungs and trachea were excised and five bronchoalveolar lavages were performed with $10 \mathrm{ml}$ of $\mathrm{NaCl} 0.9 \%$. The obtained cell suspension was centrifuged at $180 \times \mathrm{g}$ for $5 \mathrm{~min}$ and resuspended in RPMI-1640 medium containing 10\% fetal calf serum, $2 \mathrm{mM}$ glutamine, penicillin $(100 \mathrm{U} / \mathrm{ml})$ and streptomycin $(100 \mu \mathrm{g} / \mathrm{ml})$. After an attachment period of $1 \mathrm{~h}$ at $37^{\circ} \mathrm{C}$ under a gas phase of air/CO2 (95:5), the non-adherent cells were removed.

Exosome isolation. Exosomes were isolated as described $\mathrm{in}^{32}$, with some modifications. Plasma and PAAF samples $(1 \mathrm{ml})$ were centrifuged at $2,000 \times \mathrm{g}$ and $10,000 \times \mathrm{g}$ for 10 and $30 \mathrm{~min}$, respectively, at $4{ }^{\circ} \mathrm{C}$. After the 
first centrifugation step at $2000 \times \mathrm{g}$, samples were pre-treated with thrombin $(5 \mathrm{U} / \mathrm{ml})($ Sigma, St Louis, MO) for $5 \mathrm{~min}$ and centrifuged at $10,000 \times \mathrm{g}$ for $5 \mathrm{~min}$. Then, the fibrin pellet was discarded and supernatant was used for continuing the purification protocol. The $10,000 \times$ g supernatant was recovered, resuspended in $25 \mathrm{ml}$ PBS, filtered through a $0.22 \mu \mathrm{m}$ filter and ultracentrifuged at $110,000 \times \mathrm{g}$ for $70 \mathrm{~min}$. Pellet were resuspended in PBS and the remaining soluble proteins were eliminated by a final filtration through $30,000 \mathrm{mw}$ filter. Isolated exosomes were resuspended and quantified by measuring their protein content.

Nanoparticle tracking analysis. Quantification of concentration and size of exosomes obtained in plasma and PAAF were carried out using a NanoSight LM10 machine (NanoSight) by the ICTS "NANBIOSIS" at the ICMAB-CSIC. All the parameters of the analysis were set at the same values for all samples and three 1 min-long videos were recorded in all cases ${ }^{33}$. The background was measured by testing filtered PBS, which revealed no signal.

Electron microscopy. Isolated exosomes were fixed in $4 \%$ paraformaldehyde, adsorbed in formvar-coated cooper grids and negatively stained with $2 \%$ uranyl acetate. Grids were air dried and observed in a JEOL-1010 Transmission Electron Microscope ${ }^{9}$.

Exosome staining and tracking analysis. For tracking experiments, exosomes were labeled with PKH26 Red Fluorescent Cell Linker Dye (Sigma, St Louis, MO) according to the supplier's specifications. The staining reaction was stopped with 3\% BSA for $1 \mathrm{~min}$, and the labeled exosomes (Exo-PKH26) were washed three times with PBS in order to remove the unbound dye, using $300 \mathrm{KDa}$ Nanosep centrifugal devices (Pall Corporation). For each group, PBS stained with PKH26 was used as a control.

In a first tracking analysis, $7 \mu \mathrm{g}$ of Exo-PKH26 obtained from Control or AP plasma samples were resuspended in $1 \mathrm{ml}$ of saline solution and perfused through the inferior vena cava of control animals at a rate of $6 \mathrm{ml} / \mathrm{h}$ during $10 \mathrm{~min}$ as previously described ${ }^{9}$. After $30 \mathrm{~min}$, animals were sacrificed and samples of pancreas, liver, lung, kidney and small intestine were obtained and processed for the histological analysis.

In a second experiment, Exo-PKH26 from PAAF samples were perfused to control animals through the hepatic portal vein at a rate of $4 \mathrm{ml} / \mathrm{h}$ and livers from portal-perfused animals were obtained for immunofluorescence analysis.

Exosome microRNA analysis. Total RNA, including miRNAs, was isolated from exosomes using the Total Exosome RNA and Protein Isolation kit (Life Technologies, Foster City, CA, USA) according to the manufacturer's protocol. In addition, we performed an optional enrichment for small RNA during the extraction in order to obtain higher quantities of miRNAs. To monitor miRNA extraction efficiency in all samples, $5 \mu \mathrm{l}$ of synthetic $C$. elegans Cel-miR-39 ( $5 \mathrm{nM}$ ) were spiked-in in each sample before RNA extraction. We assessed exosome miRNA expression of miR-21, miR-155, miR-122 and cel-miR-39 by real-time qRT-PCR by using TaqMan microRNA reverse transcription Kit (Life technologies) according to the manufacturer's instructions. All miRNAs except the spike-in cel-miR-39 were pre-amplified. Cycle threshold $(\mathrm{Ct})$ values were calculated from automatic threshold using QuantStudioTM Real Time PCR Software. All samples showed similar extraction efficiencies according to cel-miR-39 Ct values. Due to the absence of a known endogenous miRNA in exosomes to be used for normalization, $2^{-\mathrm{Ct}}$ values were used to calculate the expression of each candidate miRNA. Values were normalized to total $\mu \mathrm{g}$ of protein present in each exosome sample.

Liquid chromatography-Mass spectrometry analysis. Protein extracts were digested with Sequencing Grade Modified Trypsin (Promega, Madison, WI) using the FASP (Filter Aided Sample Preparation) digestion protocol $^{34}$. Each tryptic peptide mixture was labeled with tandem mass tags (TMT) (Thermo Scientific, Rockford, IL) based on the standard procedure. Six labeled peptide mixtures were combined, evaporated, and desalted using a C18 SPE cartridge (Agilent Technologies, USA). Two independent TMT-labeled assays were performed $(n=4)$.

Labeled samples were analyzed by LC-MSn using an LTQ-Orbitrap XL instrument equipped with a nanoESI ion source (Proxeon, Odense, Denmark) as described ${ }^{35}$. Database search was carried using Protein Discoverer software (ThermoFisher, San Jose, CA) against the Uniprot database restricted to rat and using the target-decoy strategy. DanteR ${ }^{36}$ was used for relative quantification and statistical analysis of TMT-labeled peptides. All scans from unique peptides were considered for quantification.

Gene ontology (GO) $)^{37}$, KEGG orthology $y^{38}$ and BioGPS ${ }^{39}$ databases were used for biological categorization of the identified proteins that were significantly increased or decreased in AP conditions.

Statistical analysis. Statistical analysis was performed with GraphPad Prism software. Data are presented as mean \pm S.E.M. Data were analyzed using a two-tailed Student's t-test for comparison of two groups, and one-way analysis of variance (ANOVA) analysis followed by Tukey's post-test when comparing three groups. Statistical significance was considered when $p<0.05$.

For mass spectrometry analysis, ion reporter intensity data was normalized using QuantileN normalization. DanteR ANOVA was performed at protein level by comparing treated versus control peptides using a linear model and the $p$-values were adjusted by using the Benjamini \& Hochberg False Discovery Rate correction. Regulated peptides were determined using an adjusted $p$-value cutoff of 0.05 and a fold change lower than 0.67 (down) or higher than 1.5 (up).

Received: 13 May 2019; Accepted: 6 December 2019;

Published online: 27 December 2019 


\section{References}

1. Peery, A. F. et al. Burden and Cost of Gastrointestinal, Liver, and Pancreatic Diseases in the United States: Update 2018. Gastroenterology 156, 254-272.e11 (2019).

2. Banks, P. A. et al. Classification of acute pancreatitis-2012: revision of the Atlanta classification and definitions by international consensus. Gut 62, 102-11 (2013).

3. Singh, V. K., Moran, R. A., Afghani, E. \& de-Madaria, E. Treating acute pancreatitis: what's new? Expert Rev. Gastroenterol. Hepatol. 9, 901-11 (2015).

4. Hirota, M. et al. Relationship between plasma cytokine concentration and multiple organ failure in patients with acute pancreatitis. Pancreas 21, 141-6 (2000).

5. Gutierrez, P. T., Folch-Puy, E., Bulbena, O. \& Closa, D. Oxidised lipids present in ascitic fluid interfere with the regulation of the macrophages during acute pancreatitis, promoting an exacerbation of the inflammatory response. Gut 57, 642-8 (2008).

6. Folch, E. et al. P-Selectin Expression and Kupffer Cell Activation in Rat Acute Pancreatitis. Dig. Dis. Sci., 45, 1535-1544 (2000).

7. Freise, J., Schmidt, F. W., Magerstedt, P. \& Schmid, K. Gabexate mesilate and camostate: new inhibitors of phospholipase A2 and their influence on the alpha-amylase activity in serum of patients with acute pancreatitis. Clin. Biochem. 18, 224-9 (1985).

8. De Toro, J., Herschlik, L., Waldner, C. \& Mongini, C. Emerging Roles of Exosomes in Normal and Pathological Conditions: New Insights for Diagnosis and Therapeutic Applications. Front. Immunol. 6, 203 (2015).

9. Bonjoch, L., Casas, V., Carrascal, M. \& Closa, D. Involvement of exosomes in lung inflammation associated with experimental acute pancreatitis. J. Pathol. 240, 235-245 (2016).

10. Zhao, Y. et al. Pancreatic Acinar Cells Employ miRNAs as Mediators of Intercellular Communication to Participate in the Regulation of Pancreatitis-Associated Macrophage Activation. Mediators Inflamm. 2016, 1-11 (2016).

11. Wu, Y., Deng, W. \& Klinke, D. J. II. Exosomes: improved methods to characterize their morphology, RNA content, and surface protein biomarkers. Analyst 140, 6631-42 (2015).

12. Essandoh, K., Li, Y., Huo, J. \& Fan, G.-C. MiRNA-Mediated Macrophage Polarization and its Potential Role in the Regulation of Inflammatory Response. SHOCK 46, 122-131 (2016).

13. Liu, D. Z. et al. Elevating microRNA-122 in blood improves outcomes after temporary middle cerebral artery occlusion in rats. $J$. Cereb. Blood Flow Metab. 36, 1374-83 (2016).

14. Hsu, S.-H. et al. Essential metabolic, anti-inflammatory, and anti-tumorigenic functions of miR-122 in liver. J. Clin. Invest. 122, 2871-83 (2012)

15. Bandiera, S., Pfeffer, S., Baumert, T. F. \& Zeisel, M. B. miR-122 - A key factor and therapeutic target in liver disease. J. Hepatol. 62, 448-457 (2015).

16. Fu, D. et al. MiRNA-21 has effects to protect kidney injury induced by sepsis. Biomed. Pharmacother. 94, 1138-1144 (2017).

17. Loboda, A., Sobczak, M., Jozkowicz, A. \& Dulak, J. TGF- $\beta 1 /$ Smads and miR-21 in Renal Fibrosis and Inflammation. Mediators Inflamm. 2016, 1-12 (2016).

18. Zhou, W. et al. MicroRNA-21 down-regulates inflammation and inhibits periodontitis. Mol. Immunol. 101, 608-614 (2018).

19. Bejerano, T., Etzion, S., Elyagon, S., Etzion, Y. \& Cohen, S. Nanoparticle Delivery of miRNA-21 Mimic to Cardiac Macrophages Improves Myocardial Remodeling after Myocardial Infarction. Nano Lett. 18, 5885-5891 (2018).

20. Wan, J. et al. Inhibition of miR-155 reduces impaired autophagy and improves prognosis in an experimental pancreatitis mouse model. Cell Death Dis. 10,303 (2019).

21. Wang, Z. \& Lieberman, P. M. The crosstalk of telomere dysfunction and inflammation through cell-free TERRA containing exosomes. RNA Biol. 13, 690-695 (2016).

22. Wang, Z. et al. Telomeric repeat-containing RNA (TERRA) constitutes a nucleoprotein component of extracellular inflammatory exosomes. Proc. Natl. Acad. Sci. 112, E6293-E6300 (2015).

23. Cai, T. Q. et al. Induction of 11 beta-hydroxysteroid dehydrogenase type 1 but not -2 in human aortic smooth muscle cells by inflammatory stimuli. J. Steroid Biochem. Mol. Biol. 77, 117-22 (2001).

24. Chapman, K., Holmes, M. \& Seckl, J. 11ß-Hydroxysteroid Dehydrogenases: Intracellular Gate-Keepers of Tissue Glucocorticoid Action. Physiol. Rev. 93, 1139-1206 (2013).

25. Gulfo, J. et al. New Roles for Corticosteroid Binding Globulin and Opposite Expression Profiles in Lung and Liver. PLoS One 11, e0146497 (2016).

26. Sternby, H. et al. Determinants of Severity in Acute Pancreatitis: A Nation-wide Multicenter Prospective Cohort Study. Ann. Surg., 1 https://doi.org/10.1097/SLA.0000000000002766 (2018).

27. Aho, H. J., Suonpää, K., Ahola, R. A. \& Nevalainen, T. J. Experimental pancreatitis in the rat. Ductal factors in sodium taurocholateinduced acute pancreatitis. Exp. Pathol. 25, 73-9 (1984).

28. Folch, E., Closa, D., Prats, N., Gelpí, E. \& Roselló-Catafau, J. Leukotriene generation and neutrophil infiltration after experimental acute pancreatitis. Inflammation 22, 83-93 (1998).

29. Trush, M. A., Egner, P. A. \& Kensler, T. W. Myeloperoxidase as a biomarker of skin irritation and inflammation. Food Chem. Toxicol. 32, 143-7 (1994).

30. Shen, L., Hillebrand, A., Wang, D. Q.-H. \& Liu, M. Isolation and Primary Culture of Rat Hepatic Cells. J. Vis. Exp. https://doi. org/10.3791/3917 (2012).

31. Shelke, G. V., Lässer, C., Gho, Y. S. \& Lötvall, J. Importance of exosome depletion protocols to eliminate functional and RNAcontaining extracellular vesicles from fetal bovine serum. Journal of Extracellular Vesicles, 3, (2014).

32. Théry, C., Amigorena, S., Raposo, G. \& Clayton, A. Isolation and characterization of exosomes from cell culture supernatants and biological fluids. Curr. Protoc. Cell Biol. Chapter 3, Unit 3.22 (2006).

33. Sáenz-Cuesta, M. et al. Methods for extracellular vesicles isolation in a hospital setting. Front. Immunol. 6, 50 (2015).

34. Wiśniewski, J. R., Zougman, A., Nagaraj, N. \& Mann, M. Universal sample preparation method for proteome analysis. Nat. Methods 6, 359-62 (2009).

35. Nguyen, T. D. et al. The phosphoproteome of Human Jurkat T cell clones upon costimulation with 1 anti-CD3/anti-CD28 antibodies. J. Proteomics 131, 190-198 (2015).

36. Taverner, T. et al. DanteR: an extensible R-based tool for quantitative analysis of -omics data. Bioinformatics 28, 2404-6 (2012).

37. Gene Ontology Consortium: going forward. Nucleic Acids Res. 43, D1049-56 (2014).

38. Kanehisa, M. \& Goto, S. KEGG: kyoto encyclopedia of genes and genomes. Nucleic Acids Res. 28, 27-30 (2000)

39. Siragusa, L., Cross, S., Baroni, M., Goracci, L. \& Cruciani, G. BioGPS: navigating biological space to predict polypharmacology, offtargeting, and selectivity. Proteins 83, 517-32 (2015).

\section{Acknowledgements}

This work was supported by the projects FIS PI16/00060 and PI17/01003 from Instituto de Salud Carlos III (co-funded by FEDER-European Union) and Beca Gonzalo Miño 2017 from Asociacion Española de Gastroenterologia. The CSIC/UAB Proteomics Facility of IIBB-CSIC belongs to ProteoRed, PRB3-ISCIII, (supported by grant PT17/0019/0008). CIBEREHD is funded by the Instituto de Salud Carlos III and FEDER. 


\section{Author contributions}

E.d.-M. and D.C. conceived the experiments and analyzed data. A.J.-A., M.P.-J., L.L.-P. and L.B. carried out the experiments. J.A. and M.C. carried out the mass spectrometry analysis of exosomes. M.G. and M.M. carried out the miRNA analysis. The manuscript was written by D.C. All authors had final approval of the submitted and published versions.

\section{Competing interests}

The authors declare no competing interests.

\section{Additional information}

Supplementary information is available for this paper at https://doi.org/10.1038/s41598-019-56220-5.

Correspondence and requests for materials should be addressed to D.C.

Reprints and permissions information is available at www.nature.com/reprints.

Publisher's note Springer Nature remains neutral with regard to jurisdictional claims in published maps and institutional affiliations.

(c) (i) Open Access This article is licensed under a Creative Commons Attribution 4.0 International

License, which permits use, sharing, adaptation, distribution and reproduction in any medium or format, as long as you give appropriate credit to the original author(s) and the source, provide a link to the Creative Commons license, and indicate if changes were made. The images or other third party material in this article are included in the article's Creative Commons license, unless indicated otherwise in a credit line to the material. If material is not included in the article's Creative Commons license and your intended use is not permitted by statutory regulation or exceeds the permitted use, you will need to obtain permission directly from the copyright holder. To view a copy of this license, visit http://creativecommons.org/licenses/by/4.0/.

(c) The Author(s) 2019 